\title{
CRISP: Coarse-Grained Reconfigurable Image Stream Processor for Digital Still Cameras and Camcorders
}

\author{
Jason C. Chen and Shao-Yi Chien, Member, IEEE
}

\begin{abstract}
To design the hardware for image signal processing pipelines in digital still cameras (DSCs) and video camcoders, it is a dilemma for conventional solutions, such as application-specific integrated circuits (ASICs) and digital signal processors (DSPs), to achieve high processing capability at low cost while maintaining high flexibility for various algorithms. With the observation of the characteristics of image signal-processing pipelines, including the different requirements for different operation modes and the algorithmic similarity of image-processing tasks, a new coarse-grained reconfigurable image stream processor (CRISP) is proposed in this paper. The design idea is to devote low-cost hardware for the requirements in the preview mode and add some hardware resources for higher flexibility and processing capability in the picture-taking mode. With the coarse-grained reconfigurable stage processing elements designed for image signal-processing tasks and the reconfigurable interconnection unit with unified communication protocol, CRISP can be reconfigured as an efficient dedicated hardware in the preview mode, and it can act like a flexible DSP for the picturetaking mode with different contexts. Implementation result shows that the core (die) size is $5 \mathrm{~mm}^{2}\left(7.72 \mathrm{~mm}^{2}\right)$ with TSMC $0.18-\mu \mathrm{m}$ process, and the power consumption is $218 \mathrm{~mW}$ at $1.8 \mathrm{~V}$. At the working frequency of $115 \mathrm{MHz}$, the processor is capable of processing 11 M-pixel still images at $10 \mathrm{fps}$ for DSCs or $1920 \times 1080$ video frames at 55 fps for camcorders. CRISP can execute image pipelines 83 times faster than the state-of-the-art DSP with only about one-tenth die size.
\end{abstract}

Index Terms-Camcorder, coarse-grained reconfigurable architecture, digital still camera (DSC), hardware classification, image signal-processing pipeline.

\section{INTRODUCTION}

D IGITAL still cameras (DSCs) [1] require a significant amount of silicon components, including sensors, analog components, and digital DSC engines. Most DSCs use a CCD or CMOS imager to sense the images. After analog read-out and amplifier circuits, the sensed image data stream is then digitized and fed into the digital DSC engine, which is the brain of the camera and is responsible for performing all of the computations needed to process and compress the image. The image signal-processing pipeline is the most important

Manuscript received April 3, 2007; revised July 25, 2007. First published July 25, 2008; current version published October 8, 2008. This work is supported by VIVOTEK Inc., Chung-Ho, Taipei County, Taiwan, R.O.C. This paper was recommended by Associate Editor S. Takamura.

The authors are with Media IC and System Laboratory, Graduate Institute of Electronics Engineering and Department of Electrical Engineering, National Taiwan University, Taipei 106, Taiwan, R.O.C. (e-mail: sychien@ cc.ee.ntu.edu. tw).

Digital Object Identifier 10.1109/TCSVT.2008.928529 task executed by the digital DSC engine, since it requires high computational complexity and dominates the final image quality. As the resolution of a DSC grows to $10 \mathrm{M}$-pixel, the required computational complexity becomes enormous. Since a DSC is a computing-resource-limited embedded system, it is worthwhile to develop efficient hardware solutions for it.

Generally speaking, the DSC image pipelines contain two different modes. One is the preview mode or the camcorder mode, which has the real-time requirements for view-finding or video recording at a sufficienlty high frame rate of no less than $30 \mathrm{fps}$. The required resolution may be VGA size $(640 \times 480)$ for the small LCD screens on DSCs or full-HD size $(1920 \times 1080)$ for some high-end camcoders. To meet the real-time requirements, highly efficient hardware is usually needed for the preview/camcoder mode. The other one is the picture-taking mode, which requires more complicated processing functions in order to generate high-quality pictures. The image signal-processing pipelines in the picture-taking mode are quite different for different products of different companies. This mode does not have such crucial real-time constraints and only needs to achieve low shot-to-shot delay, which is usually one-tenth to one second, but it requires flexibility to support various and complex algorithms. Therefore, programmable hardware is usually preferred to implement the image signal-processing pipeline in the picture-taking mode in a DSC.

There are several ways to implement image pipeline algorithms in DSC systems. Some traditional solutions will be shown here, and we will also show the problems they suffer. First of all, a digital signal processor (DSP)-based solution is widely used in DSCs. Generally speaking, DSP owns high flexibility and can handle almost all kinds of image pipeline tasks in a DSC system. The drawbacks of this solution are lower efficiency and higher power consumption than dedicated hardware. Moreover, a DSP is usually unable to meet the real-time requirement in the preview mode [2], [3]. Second, application-specific integrated circuit (ASIC) is an integrated circuit which is highly specialized for a particular task or a group of related tasks. This solution is highly optimized in terms of area, power, and speed to perform its designated task. It can be designed for very fast data transfers and effective scheduling of instructions to provide high throughput. The main disadvantage of ASICs is that they typically have long design time due to the very complex design flow. They are also difficult to be reconfigured because the hardware is only optimized for a particular task. Several ASIC solutions for image pipeline are presented, such as [4]-[6]. However, it is a 


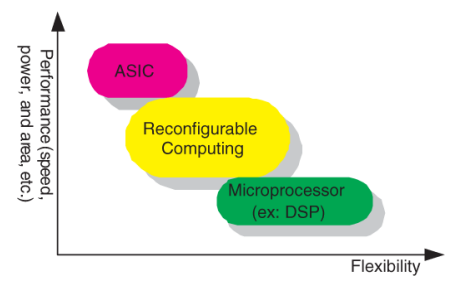

Fig. 1. Relationship among reconfigurable architecture, ASIC, and microprocessor.

dilemma to achieve high processing efficiency at low cost while maintaining flexibility and capability with the above-mentioned two conventional solutions. Therefore, a hybrid architecture is often employed, such as TI's DSC solutions [7], that is, the ASIC solution is usually adopted for the preview mode due to its high efficiency, while the DSP solution is adopted for the picture-taking mode due to its flexibility [8], [9].

On the other hand, reconfigurable computing architectures [10], where the logic gates, computing resources, and interconnections can be changed according to given configurations, seem to be a compromised solution between ASIC and DSP. The relationship among reconfigurable architecture, ASIC, and microprocessors is shown in Fig. 1. It owns high efficiency for some specific operation types and still retains enough flexibility for various application requirements by hardware reconfigurability, that is, the function of the hardware can be changed by replacing the context (or configuration) of the reconfigurable datapath and interconnection. This inspires us to combine the advantages in both ASIC-based and DSP-based solutions into a novel image processor by reconfigurable computing. That is to say, a reconfigurable image processor can be as efficient as an ASIC in the preview mode and can be flexibly reconfigured like a DSP in the picture-taking mode.

Reconfigurable hardware can be roughly classified into two types according to the granularity of the reconfigurable fabrics or processing elements (PEs) [11]: fine-grained reconfigurable processors and coarse-grained reconfigurable processors. Field-programmable gate arrays (FPGAs) [12] are one type of successful commercialized fine-grained reconfigurable computing platform. The FPGA executes a certain function by use of lookup tables (LUTs), which are essentially small local memory units where the truth tables of the desired functions are downloaded. FPGA can be used to implement any functions from bit-level. However, as the image data usually exist with a byte unit, FPGAs are not very efficient for image-processing applications. They also suffer heavy area overhead and consume a lot of power. On the other hand, coarse-grained architecture is composed with some PEs which are dedicated to some particular kinds of operations, such as addition and multiplication. It has benefits on low configuration time, low configuration memory, and low complexity of routing [13]. We think it is very suitable for image pipelines in DSC, which contain many low-level image processing functions such as 2-D filters. Coarse-grained architecture will achieve higher efficiency than FPGA and more hardware sharing capability. Several coarse-grained architectures can be found in literatures, including 1-D array architectures, such as RaPiD [14] and

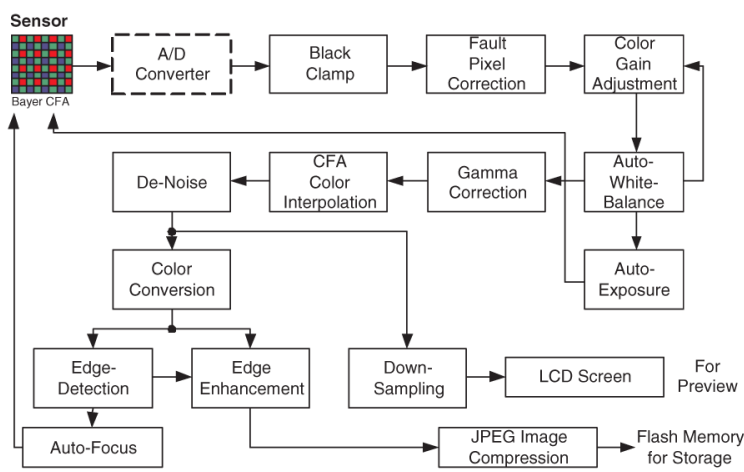

Fig. 2. Typical DSC image signal-processing pipeline, which is referred to [3].

PipeRench [15], 2-D mesh architectures, such as MATRIX [16], Garp [17], RAW [18], REMARC [19], MorphoSys [11], CHESS [20], X4CP32 [21], DRP [22], and XPP [23], and processor-tightly-coupled architecture as a reconfigurable datapath, such as PARS [24] and ADRES [25]. But all of them are not optimized for DSC and camcoder applications. Therefore, in this paper, the concept of coarse-grained reconfigurable architecture is adopted to develop a new hardware architecture for the image signal processing pipelines, which is named as coarse-grained reconfigurable image stream processor (CRISP).

The organization of the paper is as follows. In order to design a reconfigurable architecture, low-level operations in the DSC image signal-processing pipeline tasks are first analyzed and classified into some categories in Section II. After that, in Section III, every category of operations is mapped to a specific reconfigurable circuit module for some image pipeline tasks, and several modules can be combined together to finish the whole image tasks through a proposed reconfigurable on-chip interconnection. Then, in Section IV, the VLSI implementation of the proposed image stream processor is shown and compared with DSP solutions and ASIC implementations, and the flexibility of CRISP is shown in Section V. Finally, we conclude this paper in Section VI.

\section{Operation ANALYSIS AND HARDWARE ClassifiCATION OF THE IMAGE SIGNAL-PROCESSING PIPELINES}

As shown in Fig. 2, before being compressed and stored in the flash memory or displayed on the LCD screen, the sensed image data should be processed by a series of image processing procedures, which is called the DSC image signal-processing pipeline [3], [26]-[28]. The input image is first sensed by the CMOS/CCD sensor with color filter array (CFA) in Bayer pattern [29]. The image data is then read out, the dynamic range is optimized in Black Clamp, and the fault pixels are corrected. Auto-White-Balance (AWB) can estimate the color gain parameters and feedback to Color Gain Adjustment to compensate for the different illumination conditions under different light sources. Similarly, Auto-Exposure (AE) can measure the brightness of the scene and feedback to the gain control circuits and the shutter of the camera. After the image stream is processed by Gamma Correction, CFA Color Interpolation can interpolate the image in Bayer pattern, where each pixel contains only one color information $(\mathrm{R}, \mathrm{G}$, or $\mathrm{B})$, into full color resolution $(\mathrm{R}, \mathrm{G}$, and $\mathrm{B})$, and the De-Noise procedure can remove the noise in the 
image data. If the image is for the preview mode, the image data stream is then down-sampled and passed to LCD Screen. Meanwhile, the Edge Detection process is used after Color Conversion to find the edge information for Auto-Focus (AF) in $\mathrm{YCbCr}$ domain. If the image is for the picture-taking mode, the image data stream in $\mathrm{YCbCr}$ domain is enhanced by several image processing procedures, such as Edge Enhancement, and then passed to JPEG Image Compression. Finally, the compressed image data is stored in the flash memory. Note that the image signal processing pipelines for different products of different companies are quite different, including the order of the procedures, the used procedures, and the adopted algorithm of each procedure.

\section{A. Characteristics of DSC Image Signal-Processing Pipelines}

Here, some characteristics of the DSC image signal-processing pipelines are discussed. These characteristics will be considered in the design of the proposed reconfigurable processor.

1) Nonstandard: Unlike compression units defined by coding standards, there is no standard for image pipelines. Various image pipeline algorithms make traditional dedicated hardware solution out of reach for different application requirements.

2) High Computational Complexity: The computational complexity of image pipeline is proportional to the image resolution. As the resolutions of image sensors become larger and larger, the complexity becomes enormous.

3) Large Design Space: Image pipelines are composed by a series of image-processing blocks. The number of each kind of processing block, the internal functionality of each block, and the interconnection order between blocks can be modified according to different application needs.

4) Compromise of Different Design Considerations: The implementation of the DSC image signal-processing pipelines has to meet the real-time constraint in the preview mode while containing enough flexibility and capability for good quality images in the picture-taking mode. This means both efficiency and programmability are required.

5) High Resource-Sharing Potential: Processing blocks in image pipelines own high "algorithmic similarity," which means the basic operations of image pipeline procedures are similar in some specific categories, such as matrix operations or filter operations. With operational classification to group algorithms with similar computation and data access manner, hardware sharing of some specific operation types is possible.

However, conventional ASIC and DSP solutions do not readily provide for these characteristics to be taken into consideration. For a DSP-based solution, it can support large design space with its high flexibility, but it cannot meet the real-time requirements in the preview mode due to low efficiency. Moreover, the heavy computational load of image pipelines would take a long execution time and consume large power and cost by instruction execution in DSPs. For ASIC solution, it can execute dedicated tasks at low cost with high efficiency. However, its low flexibility makes it obsolete when new algorithms of image pipeline tasks are developed. In addition, hybrid architecture of DSP and ASIC may take both the advantages of high flexibility and efficiency, but the penalty is high hardware cost and low hardware utilization. The proposed coarse-grained reconfigurable architecture can contain high efficiency by hardware sharing with operational classification. A specific coarse-grained reconfigurable module is designed for each corresponding operation type, leading to efficient hardware utilization. Moreover, the reconfigurable architectures own flexibility for different algorithms in classified operation types. This can achieve low hardware cost devotion by resource-sharing. Operational analysis and classification make coarse-grained reconfigurable architecture a compromise solution, which can fit all of the characteristics of the DSC image signal-processing pipelines described above.

\section{B. Operational Classification}

Before the design of the hardware architecture, in this section, we made an algorithmic classification based on computation and data access manner of the image processing procedures. With considering the algorithmic similarity, these procedures can be grouped into several generic operation types, and the corresponding reconfigurable modules can be then designed.

1) 2-D Data Access Operation: Two-dimensional data dependency makes up a large part of image processing algorithms. When the simple image signal-processing pipeline of Fig. 2 is considered, in our profiling results on an Intel processor, it shows that more than half of the total instructions in image processing are used for data access, which means that, if we use a traditional processor architecture to implement it, there will be a great deal of waste of instruction execution for data loading and storing. Therefore, a hardware module design that efficiently supports 2-D data access in our reconfigurable processor is needed.

2) Filtering Operation: Filtering operation is also widely used in image processing. Spatial filtering is used for image smoothing, edge detection, and edge enhancement. In the spatial domain, linear image filtering can be viewed as a weighted sum of the neighboring pixels.

3) Matrix Multiplication Operation: Matrix multiplication is denoted by $\mathbf{Y}=\mathbf{A X}$, where $\mathbf{A}$ is a $m \times n$ matrix, $\mathbf{X}$ is a $n$-vector, and $\mathbf{Y}$ is a $m$-vector. In image color processing, $m$ and $n$ are usually equal to three for $\mathrm{R}, \mathrm{G}$, and $\mathrm{B}$ color components. Matrix multiplication can be used for various purposes, such as color space conversion, color-to-grayscale conversion, and color correction.

4) Pixel-By-Pixel Operation: Some image-processing algorithms are applied on the whole image, pixel by pixel. Any operations of different pixels are independent to each other, and this kind of algorithms is usually used to change general image properties. A simple datapath such as multiplication-and-accumulation (MAC) is enough for some simple operations. For some nonlinear operations, implementation with LUTs is an alternative solution. Gamma correction, brightness/contrast enhancement, and hue/saturation enhancement are good examples.

5) Statistics Gathering: The statistic property for the whole image frame is sometimes required for global image operations. The measurement parts of auto-white-balance, auto-exposure, and auto-focus belong to this operation type. The statistic of the whole image can be gathered pixel by pixel, and the gathered information is read out once when the whole image is processed. 
Therefore, statistics gathering can also be viewed as a pixel-bypixel operation.

6) Color Interpolation Operation: For CFA color interpolation, the manner of data input and output is quite different from other general image processing tasks, where the output data rate is three times of the input data rate. Moreover, different color filter arrays are used for different sensors, and there are quite a lot diverse CFA color interpolation algorithms [30]-[35]. Therefore, we classify this operation into a special type.

7) Resampling Operation: Similarly, the data access manner of resampling operation, which is often used in image scaling for display, is quite different from other operations. The output data rate is different from the input data rate, and the ratio of input/ output data rate is not fixed for different cameras. Consequently, we also put it in a special operation type.

\section{Reconfigurable Hardware Modules Based on Operation Classification}

Based on the operation classification, we proposed six coarse-grained reconfigurable hardware modules for DSC image signal-processing pipelines. Because of the algorithmic similarity between image-processing tasks, most of the operations of the pipeline can be executed efficiently with these reconfigurable modules. They can be the fabrics of the proposed coarse-grained reconfigurable image stream processor.

1) Local Memory Module: As mentioned before, memory data access consumes over half of processing instructions, which means an efficient method of data access is crucial for high-performance image processor design. Therefore, special hardware modules are devoted for fast 2-D data access and manipulation.

2) MAC Module: Most image-processing tasks are MAC-intensive operations. When the input sources of MAC modules are from 2-D windows, they can perform 2-D local MAC operations, such as image filtering. The MAC modules are designed in our processor for parallel MAC operations. The linear filtering and matrix multiplication operations can be executed in the MAC modules, and some other operations related to MAC operations can also be executed as well.

3) Pixel-Based Operation Module: Many image processing operations involve operands which are pixelwise independent. The data flow of pixel-by-pixel operations is quite simple, and the pixel-based operation modules are designed for these kind of operations. Other than the pixel by pixel operations, statistics gathering operations can also be executed in this module.

4) Color Interpolation Module: Color interpolation is one of the essential part in the DSC image pipelines. The missing color channels of images in a Bayes pattern can be generated by interpolation from neighboring color pixels. For this type of operation, we design a color interpolation module whose operands are directly from local memory modules, and different interpolation methods can be mapped.

5) Downsampler Module: Before shown on the LCD screen for preview or playback, the image with higher resolution need to be downsampled to fit to the resolution of the LCD display. The downsampler module is designed to perform fractional ratio of downsampling.
TABLE I

Devoted Hardware MOdules BaSEd ON OPERATIONAL Classification With DSC IMAGE Signal-Processing PIPELINE TASKS MAPPING

\begin{tabular}{ll}
\hline Classified Hardware Modules & Functionality \\
\hline Local Memory Module & Local data reuse and 2-D data access \\
\hline Pixel-Based Operation Module & Brightness/Contrast enhancement, \\
& Auto-white-balance, \\
& Exposure measurement, \\
& Focus value measurement, \\
& Color gain adjustment, \\
& Gamma correction \\
\hline Mul. and Acc. (MAC) Module & Edge enhancement, \\
& Anti-crosstalk, \\
& Defect pixel compensation, \\
& Color correction, \\
\hline Color Interpolation Module & Color space conversion \\
\hline Downsampler Module & Color interpolation \\
\hline ALU Module & Downsampling for LCD display \\
\hline
\end{tabular}

6) Arithmetic Logic Unit (ALU) Module: In order to increase the flexibility of our processor, ALU modules are designed for some general-purpose operations. Usually, ALU modules will be combined with other modules to perform some nonlinear image processing tasks. Nonlinear image processing is often associated with filtering techniques involving mathematical morphology, stack filters, or order statistics.

In summary, Table I shows the hardware modules and the image signal-processing tasks can be mapped for each module. Note that only a few kinds of classified modules can execute most parts of the DSC image signal-processing pipeline.

\section{Proposed CoArse-Grained ReConfigurable IMAge STREAM PROCESSOR (CRISP) ARCHITECTURE}

Here, the hardware architecture of our CRISP structure is proposed. The design concepts of CRISP are shown first followed by the detailed hardware architecture design of the processing elements and interconnection units.

\section{A. Design Ideas: Time-Space Tradeoff by Hardware Reconfiguration}

As mentioned before, the preview mode, or camcorder mode, has real-time constraints on view-finding. The picture-taking mode does not have such restricted real-time constraints but requires flexibility and capability to support various and complex algorithms. It is a dilemma for conventional solutions, such as ASIC and DSP, to achieve high processing capability at low cost while maintaining flexibility, and hybrid architecture is widely employed. Fig. 3 shows the conventional architecture of the ASIC-DSP hybrid solution [8]. In the preview mode, as shown in Fig. 3(a), a dedicated preview engine is required to meet real-time constraints since the DSP is not efficient enough. Note that the data flow from the CCD/CMOS sensor is directly passed through the preview engine and stored outside in off-chip SDRAM as the display buffer for the LCD screen. In the picture-taking mode, as shown in Fig. 3(b), there is no restricted real-time constraint, and the powerful DSP in the system can now be used to process larger image data with more complicated algorithms by executing the instructions. The image data is first read out from the sensor and stored in the off-chip SDRAM, and then the image data are processed by 


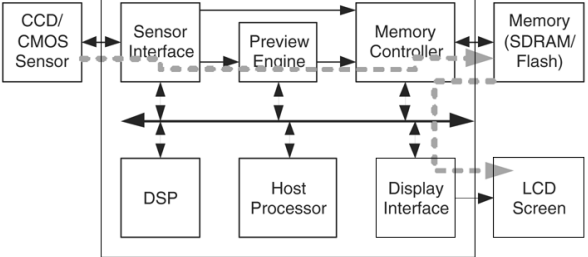

(a)

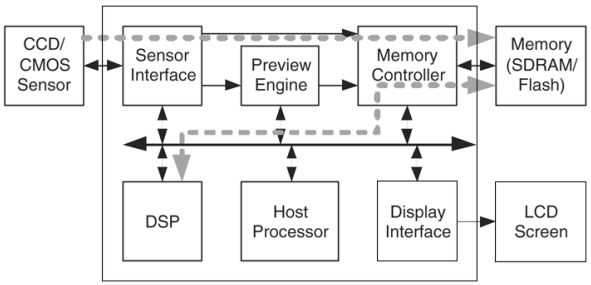

(b)

Fig. 3. Conventional ASIC-DSP hybrid architecture operating in (a) the preview mode and (b) the picture-taking mode.

the DSP. Because of the processor architecture of the DSP, at each time slot, only a small part of image data is loaded by the DSP and stored back to the SDRAM after being manipulated. It is shown that, in each mode, only one of the preview engines and the DSP are active, and the other one idles. Therefore, it is not an efficient design because of the low hardware utilization.

In this paper, CRISP is developed to achieve both the requirements in the two modes with high efficiency and high flexibility. It is based on the characteristics of the image signal-processing pipeline described in Section II. In order to achieve the two different requirements in the preview and picture-taking modes with low cost, a preview-engine-based reconfigurable architecture design concept is proposed for time-space tradeoff. Since low-level image-processing algorithms share lots of algorithmic similarity, our idea is to devote low-cost hardware-processing elements for the requirements in the preview mode and add some hardware resources to make them as reconfigurable processing elements with higher flexibility and processing capability for the picture-taking mode. In the preview mode, the image data stream is directly transferred from the image sensors and processed in real time in one pass to generate the preview video for the LCD display or video encoder, as shown in Fig. 4(a), where CRISP acts as a dedicated preview engine with a fixed context (hardware configuration). In the picture-taking mode, which has no restricted real-time constraint, the hardware can trade more execution time frames for larger image size and more complex algorithms with larger processing windows by changing the contexts for the reconfigurable hardware, as shown in Fig. 4(b). The complex image signal processing pipeline is first divided into several subpipelines. The context of each subpipeline can be loaded into CRISP to process the image data stream in one time frame, and the output image data of each subpipeline, which can be viewed as a partial result, is stored back to the off-chip SDRAM. After loading different contexts into CRISP and processing the image data stream in many time frames, the whole image signal-processing pipeline is completed, that is, CRISP acts as an image stream processor and can process the image with several passes in different time frames
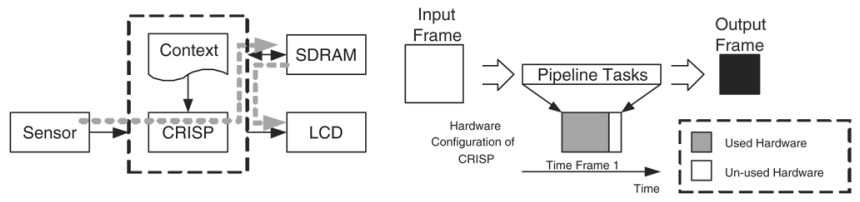

(a)
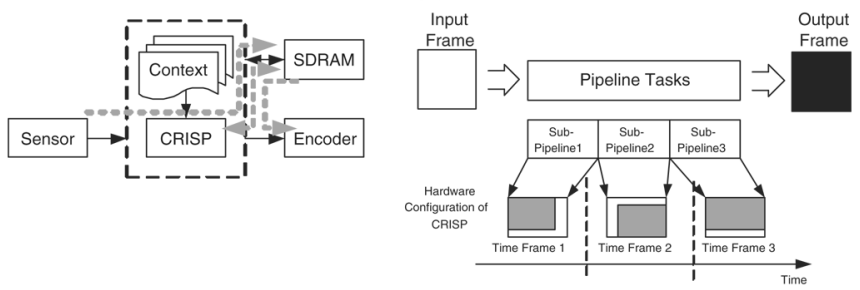

(b)

Fig. 4. Time-space tradeoff idea of CRISP operating in (a) the preview mode and (b) the picture-taking mode.

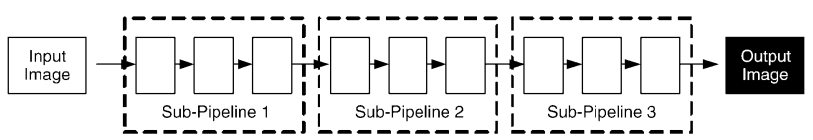

(a)

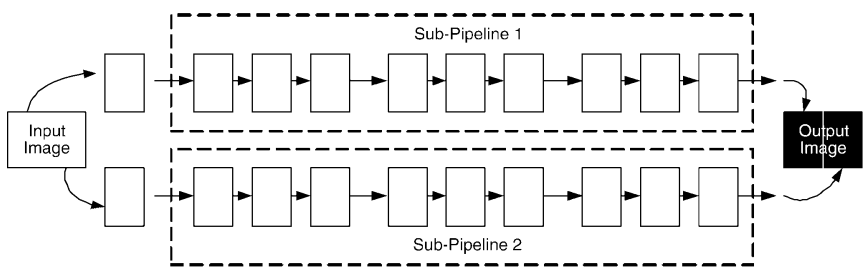

(b)

Fig. 5. Two different kinds of pipeline division. (a) Divided by functional blocks. (b) Divided on the input image.

with the off-chip memory. Note that two possible pipeline division methods can be utilized. The pipeline can be divided into subpipelines with fewer pipeline tasks, as shown in Fig. 5(a), where the context is different for each time frame. On the other hand, the image can also be divided into subimages with the same pipeline, as shown in Fig. 5(b), where the context of each time frame is the same.

The hardware architecture of CRISP is efficient. It can approach the hardware cost lower bound of the preview engine caused by the real-time constraints in the preview mode. In addition, the flexibility requirement in the picture-taking mode can be achieved by the reconfigurability of CRISP. The high processing speed can also be achieved by the processing elements specially designed for image-processing tasks by utilizing the algorithmic similarity. In summary, CRISP combines the advantages of ASIC and DSP into a single hardware by proper time-space tradeoff in different modes.

\section{B. Processor Architecture}

Here, the hardware architecture of CRISP is presented, as shown in Fig. 6, where CRISP is connected to a high-speed on-chip bus in a DSC/camcoder system-on-a-chip (SoC). It contains context registers, main controller, reconfigurable interconnection, and various kinds of coarse-grained reconfigurable stage processing elements (RSPEs). Each kind of 


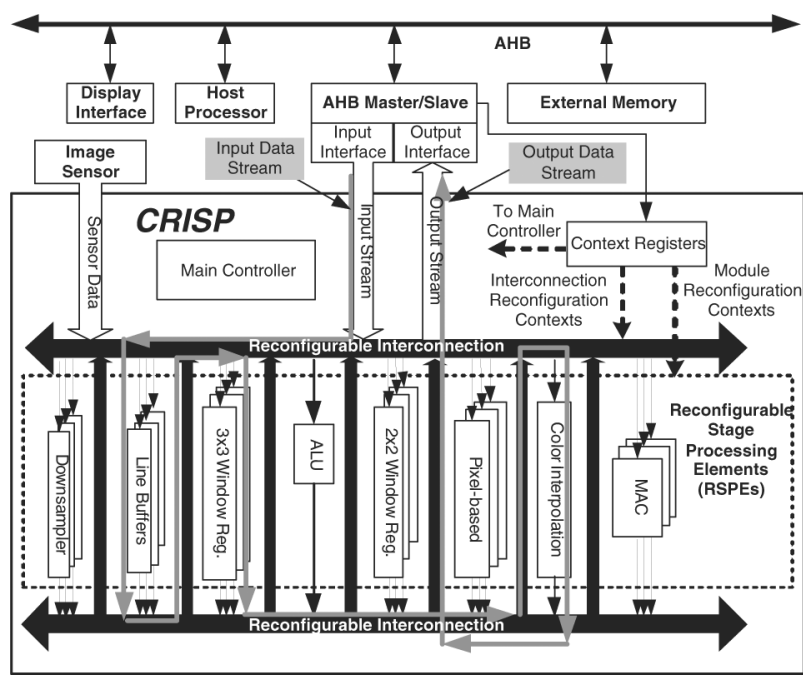

Fig. 6. CRISP processor architecture.

RSPE corresponds to one reconfigurable module described in Section II, which can be reconfigured to process some specific computations in image pipelines by operational classification in Table I. This heterogenous RSPE architecture makes the processing elements maintain high efficiency for low-level image processing. Moreover, the multiple RSPEs can be connected together to form more powerful processing pipelines through reconfigurable interconnections. For the preview mode, the input image data stream is directly from the image sensor, and the output data stream is to the external memory through the bus interface. As for the picture-taking mode, the input data stream and output data stream is from/to the external memory through the bus interface. One example is demonstrated as the gray arrow lines shown in Fig. 6. A unified communication protocol is also defined between RSPEs. This leads to the changeable interconnection orders between RSPEs for more flexible execution. In summary, the reconfigurable datapath of processing elements plus reconfigurable interconnection could enable functionalities reconfiguring within and between processing elements to achieve high flexibility.

\section{Reconfigurable Interconnection Design}

Fig. 7 shows the interconnection interface of each RSPE. Each RSPE contains an input selector, reconfigurable datapath, PE controller, stage registers, and context registers. Note that context registers of all RSPEs are connected together to the slave interface as shown in Fig. 6. All control and configuration signals are stored in context registers for input stream selection, datapath reconfiguration, and output data control. The transferred data between any two RSPEs follows a unified protocol, where both data stream and synchronization signals are contained. The $P E$ controller receives the synchronization signals from the previous stage and generates the valid signal to stage registers to control data output. Since different RSPEs may require different execution cycles for each data sample in output stream generation, and the data stream rate is bounded by the slowest one of the serial RSPEs in one time frame, the synchronization signals are used to adjust the data stream rate of different time frames. The waveforms in Fig. 8 show one example

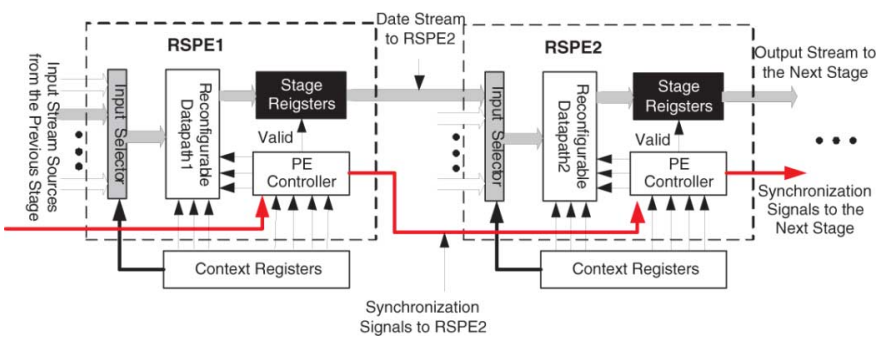

Fig. 7. Detailed architecture of the interconnection interfaces of the RSPEs.

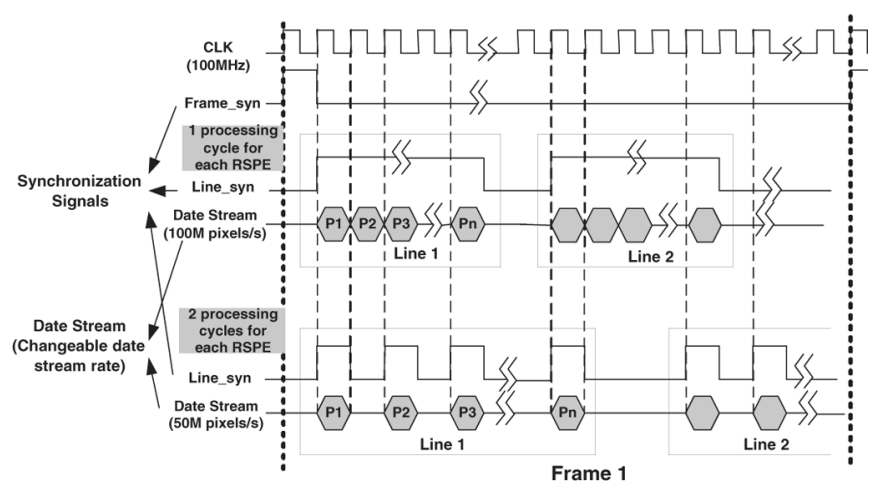

Fig. 8. Unified communication protocol for data synchronization between RSPEs.

of two different data stream rates with different synchronization signals. Two pins are used as the synchronization signals: Frame_syn is for frame synchronization, and Line_syn is for line and pixel synchronization. With the 100-MHz global clock, the data rate of the upper data stream is about $100 \mathrm{Mpixels} / \mathrm{s}$. It can be employed when all RSPEs in the pipeline can process one pixel in each cycle. If one RSPE in the pipeline requires two cycles to process one pixel, the lower one with $50 \mathrm{Mpixels} / \mathrm{s}$ can be employed instead. With this unified protocol, the data rate can be self-adjusted, and the control complexity is decreased enormously. It is the base for the design of the reconfigurable interconnection unit, and the connection order of the RSPEs can be changed without modification of the finite state machine in the main controller. Consequently, the reconfigurable interconnection unit can be simply designed with several multiplexers. Note that, because of the characteristics of image processing, these RSPEs do not have to be fully connected. For example, the input of color interpolation RSPE, which will be described in detail in Section III-D4, only connects the output of the $3 \times 3$ window register of local memory RSPE, which will be described in detail in Section III-D1. The most complex multiplexer is located in the input port of ALU RSPE, where several 11-to-1 muxtiplexers are required. The logic synthesis results show that an overhead delay of $0.29 \mathrm{~ns}$ is introduced, which is reasonably small when the target working frequency is $100 \mathrm{MHz}$. Note that ALU RSPE is designed for general purpose usage and will be described in Section III-D6.

\section{Reconfigurable Datapath Design}

Here, the reconfigurable datapath design of each kind of RSPE is presented. Each RSPE in CRISP is a coarse-grained 


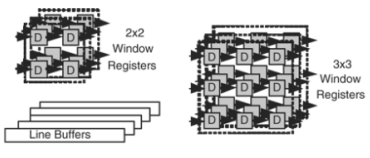

(a)

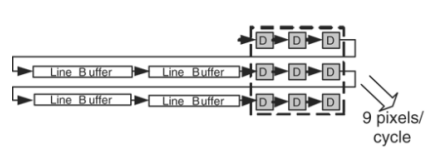

(c)

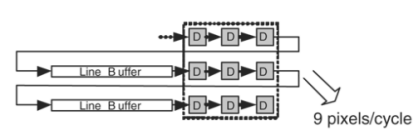

(b)

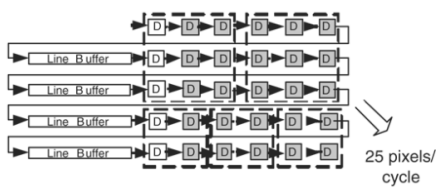

(d)
Fig. 9. (a) Local memory RSPE is composed of several $2 \times 2$ window registers, $3 \times 3$ window registers, and line buffers. (b) One configuration for $3 \times 3$ window access. (c) One configuration for $3 \times 3$ window access for images with longer width. (d) One configuration for $5 \times 5$ window access.

datapath which is responsible for some specific operations as mentioned in Section II.

1) Local Memory RSPE: For typical image signal-processing tasks, data access operations, which are not effective computations, usually occupy more than $50 \%$ of the total execution time in traditional processor architectures, and this leads to large performance degradation. In order to solve this problem, the reconfigurable memory architecture is developed for the local memory RSPE to achieve one 2-D window data access per cycle. As shown in Fig. 9(a), it is composed of several $2 \times 2$ window registers, $3 \times 3$ window registers, and line buffers, where the line buffers are often implemented with on-chip dual-port SRAM with an address counter. By combining two line buffers and one $3 \times 3$ window registers as Fig. 9(b), nine pixels of a $3 \times 3$ window can be accessed per cycle with an input image stream in raster-scan manner, which is the delay line architecture widely used in image processing VLSI [36]. Similarly, several basic windows and lines can be clustered for more complicated configurations. For image with longer width, several line buffers can be connected serially as shown in Fig. 9(c). If the image-processing task requires larger window data, several windows and lines can be connected as shown in Fig. 9(d), where $5 \times 5$ window data can be accessed in each cycle by activating 25 output channels of the $5 \times 6$ window registers. Note that the combining of these components is done by changing the interconnection with several multiplexers, which are not shown in these figures for simplification. Also note that, in local memory RSPE, the length of the delay lines can be reconfigured by concatenating several short line buffers or changing the boundary of the address counters of long line buffers.

There is a tradeoff between chip area and power for the selection of the length of the line buffers. For a 1920-pixel line, several different configurations can be chosen. It can be composed of one 1920-pixel line buffer, two 960-pixel line buffers, or four 480-pixel line buffers. Referring to the on-chip memory library provided by Artisan for TSMC 0.18 $\mu \mathrm{m}$ process, we can find that the configuration of small line buffers consumes less power with larger area. The configuration of one 1920-pixel line buffer requires power consumption of $11.40 \mathrm{~mW}$ with the area of $0.37 \mathrm{~mm}^{2}$. The configuration of two 960-pixel line buffers requires power consumption of 9.93

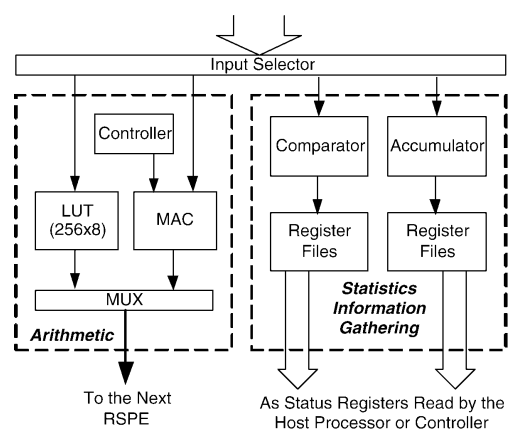

Fig. 10. Architecture of pixel-based RSPE.

$\mathrm{mW}$ with the area of $0.42 \mathrm{~mm}^{2}$. The configuration of four 480-pixel line buffers requires power consumption of $9.20 \mathrm{~mW}$ with the area of $0.53 \mathrm{~mm}^{2}$. The optimal solution depends on the design target. If the target width for preview is less than 960 pixels and hardware cost is the major concern, 960-pixel line buffers are employed because they can support the shortest line requirement with the more cost-efficient configuration.

2) Pixel-Based Operation RSPE: The architecture of pixelbased operation RSPE is shown in Fig. 10. This module is designed for operations whose data flow is pixel by pixel. We separate the pixel-based operation RSPE by two main parts. One is for arithmetic operations, such as pixel by pixel multiplication or addition, and the other one is for statistics information gathering. The arithmetic part in each pixel-based RSPE is composed of one MAC unit with its controller for conditional MAC operations on each input pixel, a LUT, and a multiplexer between the MAC and the LUT for internal communication. With these components, most of the pixel-by-pixel image-processing tasks can be executed, such as brightness/contrast enhancement, saturation enhancement, color gain adjustment, and gamma correction. Some image-processing algorithms require statistics information of the whole image, such as white point detection and luminance of some specified regions, and this is also done in the pixel-based operation RSPE. The accumulator and comparator help to gather necessary information in the input image stream and store it to the register files, which are connected to the bus interface of CRISP and can be read by the host processor after the data stream of the whole image is processed. The host processor can utilize the information in register files to determine the proper parameters for upcoming input data. Several auto-white-balance, auto-exposure, and auto-focus operations can be implemented with these hardware components.

3) MAC RSPE: MAC RSPEs are used to handle 2-D filters and matrix operations. It is not a simple MAC but is a general processing element for many kinds of 2-D image filters. The input sources of the MAC RSPE are mainly from $3 \times 3$ window registers for 2-D filters or from the three color component of one pixel for matrix operations. As shown in Fig. 11(a), there are nine functional units (FUs) in each MAC RSPE for the nine registers of a $3 \times 3$ window register, and each $\mathrm{FU}$ can generate its own outputs as a multiplier, adder, subtractor, or differencer. The FU can also be connected to perform matrix operations, such as color space conversion. The adder tree is used to sum up the products of each FU for 2-D linear filters. A sorter is embedded in the MAC RSPE for order filters, such as median 


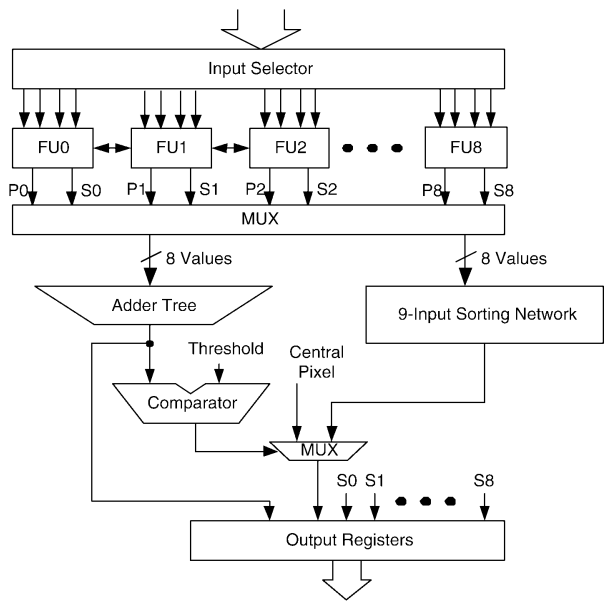

(a)

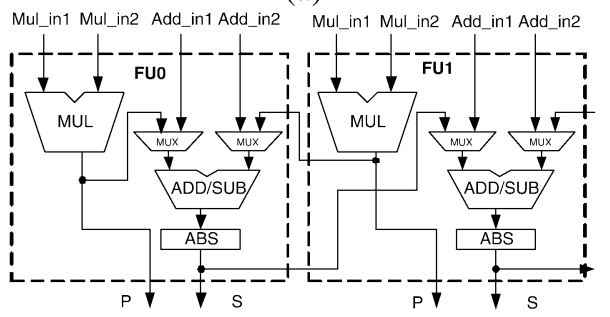

(b)

Fig. 11. (a) Hardware architecture of MAC RSPE. (b) Architecture of the internal FUs.

filters and morphology filters. In addition, a comparator is also designed to support some nonlinear operations. The architecture of each FU and the interconnection between FUs are shown in Fig. 11(b). Each FU contains one multiplier and one adder/subtractor with absolute operator, and the output of each FU can be selected from them, which is $\mathrm{P}$ or S. Moreover, the FUs can also be connected to their neighbors serially for more complex operations. Note that some multiplexers for reconfiguration are not shown in these figures for simplification. For example, the absolute operator can be bypassed by a multiplexer not shown in the figure.

4) Color Interpolation RSPE: Color interpolation RSPE is designed to handle CFA color interpolation. Fig. 12(a) shows the top view of this module, and at most four window registers can be the input of the color interpolation RSPE to support complex CFA color interpolation algorithms. Every block in Fig. 12(a) represents an FU shown in Fig. 12(b). C and D in Fig. 12(a) represent cross and diagonal named by the cross data and diagonal data of the input $3 \times 3$ window registers, that is, the FUs $\mathrm{C} 1$ and D1 can access the cross data and diagonal data of $3 \times 3$ window register 1. Similarly, C2, D2, C3, D3, C4, and D4 can access data in the rest three window registers. The outputs of $\mathrm{C} 1$ and $\mathrm{C} 2$ are sent to $\mathrm{C} 12$, and it is similar for D12, C34, and D34. In the third stage, the inputs of $\mathrm{C} 1234$ are from $\mathrm{C} 12$ and C34, and those of D1234 are from D12 and D34. Finally, the outputs of C12, D12, C34, D34, C1234, and D1234 can be sent to the registers in the ALU RSPE described in Section III-D6, where some complicated interpolation algorithms can be performed and the interpolated RGB data can be generated. Note

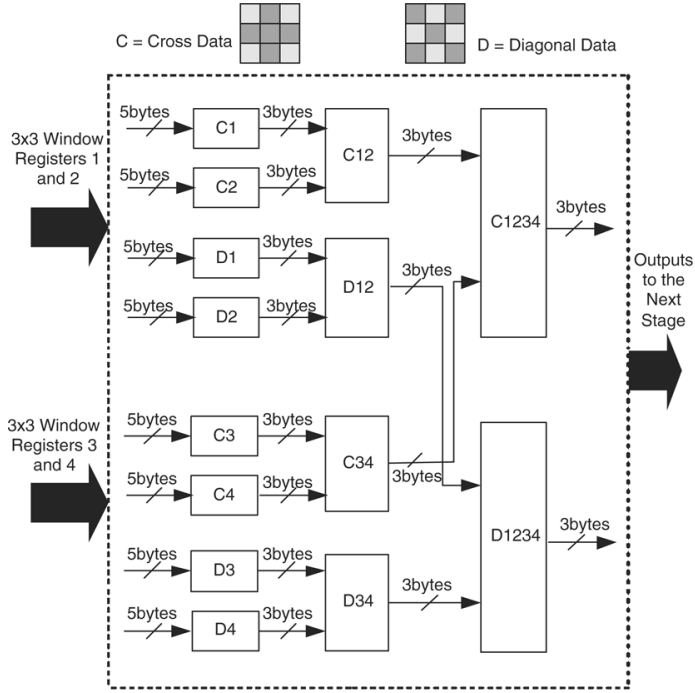

(a) From Memory Modules / Outputs of the Last Stage FUs

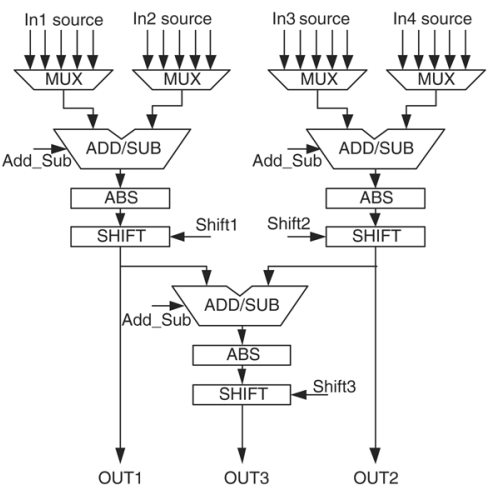

(b)

Fig. 12. (a) Color interpolation RSPE and (b) its internal FUs.

that the cross data and diagonal data are processed separately in this RSPE since from the observation of existing CFA color interpolation algorithms, the cross data and diagonal data are often processed separately because of the nature of the Bayer CFA pattern.

5) Downsampler RSPE: The downsampler RSPEs are required for image scaling for previewing on LCD display. They can support a fractional ratio. Four-point bilinear interpolation is employed so one line buffer is required for one color channel downsampling.

6) ALU RSPE: In order to increase the flexibility and processing capability, a dedicated ALU RSPE for general purpose operations is designed, and it can be combined with other RSPEs to enable more complex operations. Fig. 13(a) shows the architecture of an ALU module, and the hardware architecture of its internal FUs is shown in Fig. 13(b). The ALU RSPE is composed of four FUs in parallel or serial connection. The output of each FU can be stored in one of the registers in the register file of ALU RSPE, and the stored values in these registers can be the inputs in the next cycle. The input sources of ALU RSPE can be from other RSPEs in the processor for further data manipulation. Each FU in this RSPE can also directly access one 


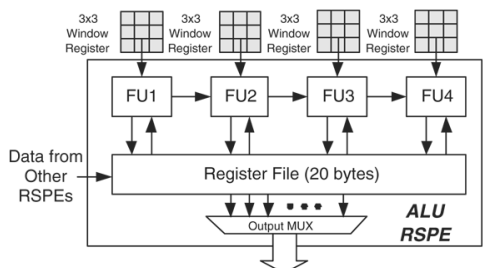

(a)

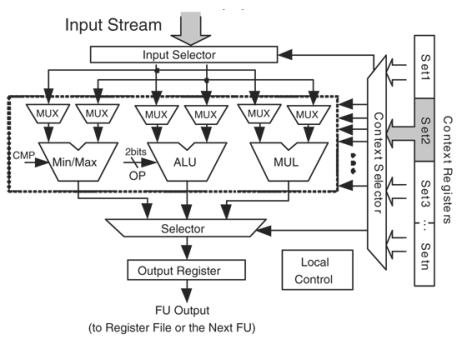

(b)

Fig. 13. Architecture of (a) the ALU RSPE and (b) the internal FU.

corresponding $3 \times 3$ window register for fast window data manipulation. Several context register sets can be used in the ALU RSPE, which can be viewed as the microcode for the ALU for more complex operations, and they can also be employed for location-dependent operations. One example is the CFA color interpolation operations for Bayer-patterned image data, where the operations for R, G, and B pixels at different locations are different, and different context sets are required.

\section{E. Control and Configuration Mechanism}

CRISP can work with simple control and configuration mechanism. As shown in Fig. 6, it can be controlled through the slave interface by the host processor, and all the control registers, context registers, and LUTs are connected to the slave interface. First of all, CRISP is idled, and the input interfaces from the bus and image sensor are turned off by the Main Controller. The host processor then moves context data (configurations) from the external memory to Context Registers to reconfigure the Reconfigurable Interconnection and all RSPEs. Next, CRISP is activated, and Main Controller activates the input interface, which can access image data from image sensor or from external memory via master interface to form Input Data Stream following the protocol described in Section III-C. With Reconfigurable Interconnection, the data stream is processed by several RSPEs to generate Output Data Stream, which is sent to the external memory by the master output interface. After the whole image is processed, in preview mode, the output image is then displayed on the LCD. On the other hand, in picture-taking mode, several iterations (time frames) are required to generate one high-quality large output image.

\section{VLSI IMPLEMENTATION OF CRISP AND COMPARISON}

A prototype chip of CRISP was implemented in real silicon. Here, the details of the implementation results are shown and compared with existing DSP solutions and ASIC implementations.
TABLE II

GATE COUNTS OF EACH KIND OF RSPES IN CRISP

\begin{tabular}{lcc}
\hline Hardware Modules & Gate Counts & Percentage (\%) \\
\hline Window registers & 7,880 & $4.6 \%$ \\
Color interpolation & 17,424 & $10.1 \%$ \\
ALU & 37,956 & $22.2 \%$ \\
MAC (x3) & 43,681 & $25.5 \%$ \\
Pixel-Based Operation (x3) & 22,193 & $13 \%$ \\
Downsampler (x3) & 24,456 & $14.3 \%$ \\
Control \& Others & 17,649 & $10.3 \%$ \\
\hline Total & 171,239 & $100 \%$ \\
\hline
\end{tabular}

TABLE III

CRISP CHIP SPECIFICATIONS

\begin{tabular}{lc}
\hline Technology & TSMC $0.18 \mu \mathrm{m}$ CMOS 1P6M \\
Supply Voltage & $1.8 \mathrm{~V}$ \\
Core Size & $2.24 \times 2.24 \mathrm{~mm}^{2}$ \\
Logic Gates (2-input NAND gate) & $171.2 \mathrm{k}$ \\
On-chip SRAM & $9,568 \mathrm{bytes}$ \\
Max Operating Frequency & $115 \mathrm{MHz}$ \\
Max Input Date Rate (1 pixel =8 bits) & $115 \mathrm{Mpixels} / \mathrm{s}$ (Preview) \\
& 345Mpixels/s (Picture-taking) \\
Max Output Date Rate & $345 \mathrm{Mpixels} / \mathrm{s}$ \\
Max Computation Performance & $13.05 \mathrm{G}$ fixed point ops./s \\
Max Video Processing Capability & $1920 \times 1080(4: 4: 4), 55 \mathrm{frames} / \mathrm{s}$ \\
Max Image Processing Capability & $4072 \times 2720$ (Full Color), 10frames/s \\
Power Consumption & $218 \mathrm{~mW} @ 115 \mathrm{MHz}$ \\
\hline
\end{tabular}

\section{A. Implementation Results}

CRISP was implemented with cell-based design flow with Verilog-HDL. Table II shows the logic gate count of each hardware module synthesized with Synopsys Design Vision. The total logic gate count is about $171 \mathrm{~K}$. The gate count of context registers are included in each individual RSPE, respectively, and the line buffers are implemented with on-chip SRAM, which are not included in this table. Note that several RSPEs have three copies in this prototype chip, such as MAC RSPE, Pixel-Based Operation RSPE, and Downsampler RSPE. These RSPEs are marked with " $x 3$ " in this table. The number of RSPEs is configurable before fabrication and is usually determined by the real-time requirement of the preview mode. The chip was successfully fabricated with TSMC $0.18-\mu \mathrm{m}$ 1P6M CMOS process with correct measurement results. The chip specifications are shown in Table III, and the chip micrograph is illustrated in Fig. 14. The chip die size is $2.78 \mathrm{~mm} \times 2.78 \mathrm{~mm}$, and the core size is $2.24 \mathrm{~mm} \times 2.24 \mathrm{~mm}$. The total on-chip SRAM bit number is $76544 \mathrm{~b}$. CRISP can have two different working clock rates, which are the internal clock rate and sensor data rate, respectively. With a maximum working frequency of $115 \mathrm{MHz}$, the maximum input data rate in the preview mode is $115 \mathrm{Mpixels} / \mathrm{s}$ and $345 \mathrm{Mpixels} / \mathrm{s}$ in the picture-taking mode. The maximum computation capability is $13050 \mathrm{MOPS}$ at $115 \mathrm{MHz}$. For highdefinition $(H D)$ video $(1920 \times 1080)$ in $4: 4: 4$ format, CRISP can achieve the processing speed of 55 frames/s. As for images with 11 Mpixels, the processing speed of 10 frames/s can be also achieved. It shows that CRISP can meet the requirements of high-end video camcoders and DSCs with the power consumption of $218 \mathrm{~mW}$.

\section{B. Comparison With Conventional Solutions}

As mentioned in Section I, DSPs and ASICs are the two most practical and popular solutions to implement image signal pro- 


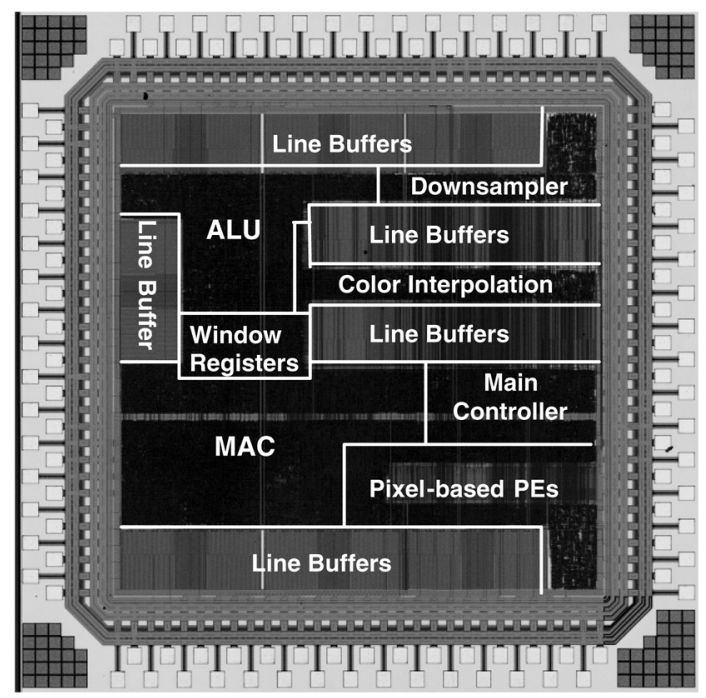

Fig. 14. Chip micrograph of CRISP.

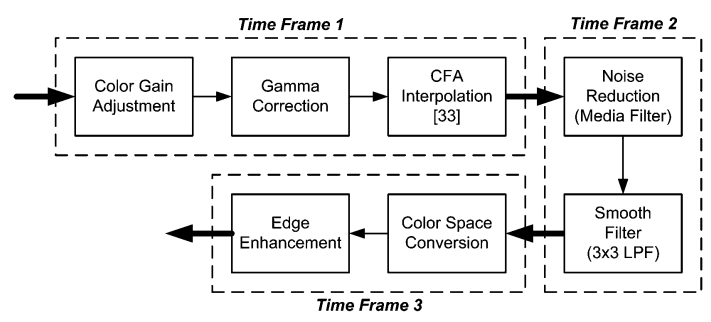

Fig. 15. Test image pipeline for the comparison.

cessing pipelines, especially for high resolution applications. Thus, in this section, we compare our work with DSPs and ASICs, respectively, to show the advantages of it.

1) Comparison With Digital Signal Processors (DSPs): The image signal-processing pipeline in Fig. 15 is used as the testbench for the comparison, and CRISP is compared with two high-end commercial DSPs, Trimedia TM1300 [37] and TI TMS320C64x [38]. The pipeline in Fig. 15 is mapped to CRISP with three subpipelines in three different time frames at $100-\mathrm{MHz}$ working clock rate. The input is 8 -b image data in a Bayer pattern. In the first time frame, the input data rate is $50 \mathrm{MB} / \mathrm{s}$ since the more complicated color interpolation method is adopted, where two-cycle latency is required for each pixel. After the first time frame, CRISP is reconfigured for different image pipeline tasks in the second time frame. The input data rate now is $300 \mathrm{MB} / \mathrm{s}$, that is, CRISP can process one pixel in RGB 24-bit format every cycle. In the last time frame, CRISP is again reconfigured for color space conversion and edge enhancement operations, and the input data rate is also $300 \mathrm{MB} / \mathrm{s}$.

Table IV and Fig. 16 show the execution time comparison with TM1300 and TMS320C64x on the same image signal pipeline tasks. The test image size is $4072 \times 2720$. It shows that, when executing the whole image pipeline, CRISP is over 276 times and 83 times faster than TM1300 and TMS320C64x, respectively. The large 2-D image access latency of DSPs is hidden by specially designed hardware modules in CRISP. Moreover, the reconfigurable modules with reconfigurable

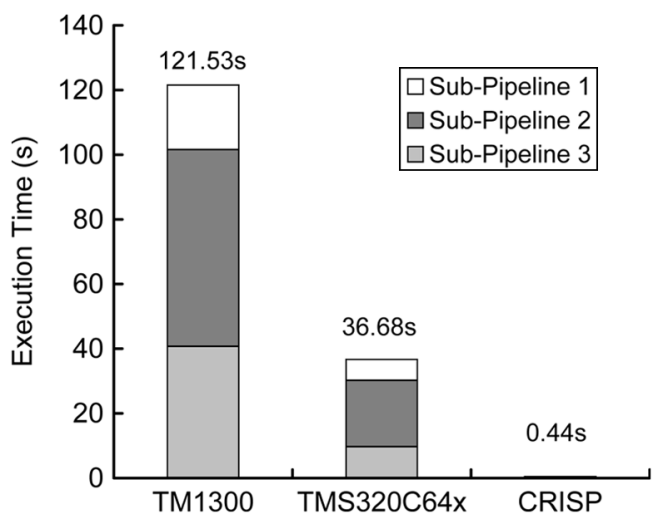

Fig. 16. Execution time ratio of the image pipeline in Fig. 15.

TABLE IV

EXECUTION TIME COMPARISON WITH TM1300 AND TMS320C64X OF IMAGE PIPELINE IN Fig. 15

\begin{tabular}{|c|c|c|c|c|}
\hline \multirow[b]{2}{*}{ Sub-Pipeline } & \multirow[b]{2}{*}{ Contained Tasks } & \multicolumn{3}{|c|}{ Execution Time } \\
\hline & & $\begin{array}{c}\text { TM1300 } \\
{[37]}\end{array}$ & $\begin{array}{c}\text { TMS320C64x } \\
{[38]}\end{array}$ & CRISP \\
\hline $\begin{array}{l}\text { Sub-Pipeline } 1 \\
\text { (Time Frame 1) }\end{array}$ & $\begin{array}{l}\text { White balance } \\
\text { Gamma correction } \\
\text { Color interpolation }\end{array}$ & $19.97 \mathrm{~s}$ & $6.44 \mathrm{~s}$ & $0.22 \mathrm{~s}$ \\
\hline $\begin{array}{l}\text { Sub-Pipeline } 2 \\
\text { (Time Frame 2) }\end{array}$ & $\begin{array}{l}\text { Noise reduction } \\
\text { Smoothing filter }\end{array}$ & $60.83 \mathrm{~s}$ & $20.55 \mathrm{~s}$ & $0.11 \mathrm{~s}$ \\
\hline $\begin{array}{l}\text { Sub-Pipeline } 3 \\
\text { (Time Frame 3) }\end{array}$ & $\begin{array}{l}\text { Color space conversion } \\
\text { Edge enhancement }\end{array}$ & $40.73 \mathrm{~s}$ & $9.69 \mathrm{~s}$ & $0.11 \mathrm{~s}$ \\
\hline Whole Pipeline & & $121.53 \mathrm{~s}$ & $36.68 \mathrm{~s}$ & $0.44 \mathrm{~s}$ \\
\hline
\end{tabular}

interconnection design provide large computation ability to support a high data input rate. These advantages of CRISP lead to apparent execution speedup with respect to DSP architectures. It is also shown in Table IV that, no matter how many tasks there are, the execution time of CRISP is only related to the number of time frames and the latency to process each pixel.

The silicon comparisons with Trimedia TM1300 and TI TMS320C64x are shown in Table V. Compared with the DSP solutions, the on-chip memory size of CRISP is reduced dramatically since no instruction memory and cache memory are required, and it only needs the small-sized microcodes stored in context registers. Experimental results show the proposed processor achieves better area and power efficiency indexes defined as the following equations, where lower is better:

Cost Efficiency $=($ Normalized die size $) \times($ Execution time $)$

Energy $=($ Power consumption $) \times($ Execution time $)$

where the data of execution time are from Table IV. In summary, compared with TMS320C64x, CRISP is 83 times faster with only one-tenth die size even with a less advanced process.

2) Comparison With ASICs: We also compare CRISP with ASIC solutions to show the overhead of flexibility. Two image signal-processing pipelines are considered. The first one is shown in Fig. 17, which is also a simplified image pipeline for preview mode. The image size is set as $800 \times 600$. The 
TABLE V

SILICON COMPARISON WITH TRIMEDIA TM1300 AND TI TMS320C64X

\begin{tabular}{|c|c|c|c|}
\hline Index & $\begin{array}{c}\text { TM1300 } \\
{[37]}\end{array}$ & $\begin{array}{c}\text { TMS320C64x } \\
{[38]} \\
\end{array}$ & CRISP \\
\hline Technology & $0.35 \mu \mathrm{m}$ & $0.13 \mu \mathrm{m}$ & $0.18 \mu \mathrm{m}$ \\
\hline Die Size & $58 \mathrm{~mm}^{2}$ & $72 \mathrm{~mm}^{2}$ & $7.72 \mathrm{~mm}^{2}$ \\
\hline $\begin{array}{l}\text { Normalized Die size } \\
\text { (to } 0.18 \mu \mathrm{m} \text { ) }\end{array}$ & $14.5 \mathrm{~mm}^{2}$ & $144 \mathrm{~mm}^{2}$ & $7.72 \mathrm{~mm}^{2}$ \\
\hline Frequency & $200 \mathrm{MHz}$ & $600 \mathrm{MHz}$ & $115 \mathrm{MHz}$ \\
\hline Power Consumption & 3,200mW@2.5V & 718mW@1.2V & 218mW@1.8V \\
\hline On-chip SRAM & $48 \mathrm{kB}$ & $1 \mathrm{MB}$ & $9 \mathrm{kB}$ \\
\hline Cost Efficiency $\left(\mathrm{mm}^{2} * \mathrm{~s}\right.$ & $1.76 \mathrm{k}$ & $5.28 \mathrm{k}$ & $0.0034 \mathrm{k}$ \\
\hline $\begin{array}{l}\text { Cost Efficiency } \\
\text { (Excluded Memory) }\end{array}$ & N/A & $2.65 \mathrm{k}^{*}$ & $0.0021 \mathrm{k}$ \\
\hline Energy $(\mathrm{mW} * \mathrm{~s})$ & $388.90 \mathrm{k}$ & $26.34 \mathrm{k}$ & $0.10 \mathrm{k}$ \\
\hline $\begin{array}{l}\text { Normalized Energy } \\
\left(\mathrm{mW} * \mathrm{~s}^{* *}\right.\end{array}$ & $97.22 \mathrm{k}$ & $52.67 \mathrm{k}$ & $0.10 \mathrm{k}$ \\
\hline $\begin{array}{r}* \text { The die size without } \\
* * \text { The ener }\end{array}$ & $\begin{array}{l}\text { mory is estima } \\
\text { is normalized }\end{array}$ & $\begin{array}{l}\text { from the die } \mathrm{p} \\
0.18 \mu \mathrm{m} \text { process }\end{array}$ & \\
\hline $\begin{array}{l}\text { From } \\
\text { CCD } \rightarrow \text { White } \\
\text { Sensor }\end{array}$ Balance $\rightarrow$ Inter & $\rightarrow \begin{array}{c}\text { Color } \\
\text { Correction }\end{array}$ & $\begin{array}{c}2-D \\
\text { Filtering }\end{array} \rightarrow \begin{array}{r}\text { Down- } \\
\text { sampling }\end{array}$ & $\rightarrow \stackrel{\text { LCD }}{\text { LCD }}$ \\
\hline
\end{tabular}

Fig. 17. Simple image signal-processing pipeline for preview mode in a DSC.

TABLE VI

IMPLEMENTATION COMPARISON WITH ASIC

\begin{tabular}{lcccc}
\hline Index & ASIC & CRISP & ASIC & CRISP \\
& (Preview) & (Preview) & (Picture-Taking) & (Picture-Taking) \\
\hline Logic Gate Count & 76,386 & 171,239 & 229,195 & 171,239 \\
Memory (Bytes) & 9,568 & 9,568 & 22,656 & 9,568 \\
Equivalent Total & 306,018 & 400,871 & 772,939 & 400,871 \\
Gate Count & & & & \\
Processing Time (s) & 0.0048 & 0.0048 & 0.11 & 0.44 \\
Cost Efficiency* & 1,469 & 1,924 & 85,023 & 176,383 \\
\hline
\end{tabular}

* Lower is better.

other one is the image-processing pipeline shown in Fig. 15, which is a simplified image pipeline for picture-taking mode with the resolution of $4072 \times 2720$. Note that, although the pipelines in Figs. 15 and 17 are simplified versions and some important operations are not included, they can be the test examples for the comparison of hardware efficiency. For fair comparison, the two pipelines are implemented in ASIC with the same design style by ourselves, and the results are shown in Table VI, where the index "Cost Efficiency" is defined as (1), where total gate count is used instead of die size here. For the preview mode, we set the input data rates of both ASIC and CRISP as 100 Mpixels/s. It shows that the overhead for the reconfigurability is about $30 \%$ compared with the dedicated implementation, and the cost efficiency of CRISP is close to that of ASIC. As for the picture-taking mode, we set input data rates of both ASIC and CRISP as 300 Mpixels/s. The ASIC is designed to process the image with one pass (time frame), while CRISP requires several passes since the hardware resource is limited and shared in temporal. Presumably, the cost efficiency is worse than that of ASIC because of the lower hardware utilization, and the hardware cost (gate count) is much less because of the time-space tradeoff concept. It is also shown in this experiment that CRISP can meet the requirements of different modes with the same hardware.

\section{Comparison With Advanced Image/Video Processors}

We also compare CRISP with several advanced image/video processors in this subsection. Here, two types of image/video processors are compared: single-instruction-multiple-data (SIMD) machines and stream processors. Several SIMD machines have been proposed, such as IMAP [39] and XETAL-II [40]. They are often used for vision applications, such as scene analysis for intelligent transportation systems. SIMD machines are usually composed of large amount of processing elements working in SIMD manner and can often provide high computing power, which can be more than 100 G-operations per second (GOPS) [40]. However, high-bandwidth input/output channels are usually required, and they also have large power consumption and hardware cost. For DSC applications, where the bandwidth of input channel is often 1 pixel per cycle and power consumption and hardware cost are main design issues, SIMD machines are not suitable. It is an overkill solution for DSC.

On the other hand, stream processors, such as Imagine [41] and SPI [42], have proved to be efficient solutions for mediaprocessing applications. The key idea of stream processors is to organize an application into streams and kernels. The data is formatted as stream with elements in uniform data structure, and the kernel is the process described by a program iteratively executed for each element. Stream processors can usually achieve high performance for media applications by employing locality and concurrency, and complex SIMD kernel executing unit and large stream register file are usually employed. Similarity, existing stream processors [41], [42] are also overkill for DSC applications. CRISP can also be viewed as a special optimized case of stream processor. In CRISP, the kernel executing unit is the heterogeneous RSPE, where the kernel is executed by a configured hardware instead of executing programs. Besides, no stream register file is needed because of the regular and pipelined processing, and high computing power can still be achieved with task pipelining by several RSPEs connected as a chain.

\section{FLEXIBILITY OF CRISP}

In addition to high performance, CRISP also has the advantages of high flexibility. Both online and offline flexibility are supported by CRISP. Offline flexibility, or architecture scalability, means the flexibility before fabrication. Designers can configure the number of each kind of RSPE before fabrication to meet different processing speed and hardware cost requirements for different applications. After fabrication, the number of RSPEs cannot be changed. Designers can change the context to reconfigure the operations of each RSPE and the interconnection between RSPEs to perform different image-processing procedures as DSP and FPGA.

\section{A. Online Flexibility: Color Interpolation Examples}

In order to demonstrate the flexibility of the CRISP architecture, we try to map many different image processing algorithms on CRISP. As mentioned before, color interpolation is one of the most essential and flexible processing block in image pipelines. In this section, we take color interpolation as an example and map four well-known color interpolation methods, which are bilinear [30], edge sensing [31], linear interpolation with Laplacian second-order correction terms [32], and effective color interpolation using signal correlation 
TABLE VII

HARDWARE MAPPING ON CRISP FOR BILINEAR, EDGE SENSING, LAPLACIAN, AND ECI

\begin{tabular}{|c|c|c|c|}
\hline Algorithm & $\begin{array}{l}\text { Required Hardware } \\
\text { Resource }\end{array}$ & $\begin{array}{l}\text { Critical } \\
\text { Module } \\
\text { Latency }\end{array}$ & $\begin{array}{l}\text { Max Output } \\
\text { Data Rate }\end{array}$ \\
\hline $\begin{array}{l}\text { Bilinear } \\
{[30]}\end{array}$ & $\begin{array}{c}\text { (Line buffers) } \times 2 \\
(3 \times 3 \text { window }) \times 1 \\
\text { Color Interpolation RSPE }\end{array}$ & 1 & 345M pixels/s \\
\hline $\begin{array}{l}\text { Edge sensing } \\
\text { [31] }\end{array}$ & $\begin{array}{c}\text { (Line buffers) } \times 2 \\
(3 \times 3 \text { window }) \times 1 \\
\text { Color Interpolation RSPE } \\
\text { ALU RSPE }\end{array}$ & 1 & $345 \mathrm{M}$ pixels $/ \mathrm{s}$ \\
\hline $\begin{array}{l}\text { Laplacian } \\
\text { [32] }\end{array}$ & $\begin{array}{c}\text { (Line buffers) } \times 4 \\
(3 \times 3 \text { window }) \times 2 \\
\text { Color Interpolation RSPE } \\
\text { ALU RSPE }\end{array}$ & 1 & $345 \mathrm{M}$ pixels $/ \mathrm{s}$ \\
\hline $\begin{array}{l}\text { ECI } \\
{[33]}\end{array}$ & $\begin{array}{c}\text { (Line buffers) } \times 7 \\
(3 \times 3 \text { window }) \times 4 \\
(2 \times 2 \text { window }) \times 2 \\
\text { Color Interpolation RSPE } \\
\text { ALU RSPE }\end{array}$ & 2 & $172 \mathrm{M}$ pixels/s \\
\hline
\end{tabular}

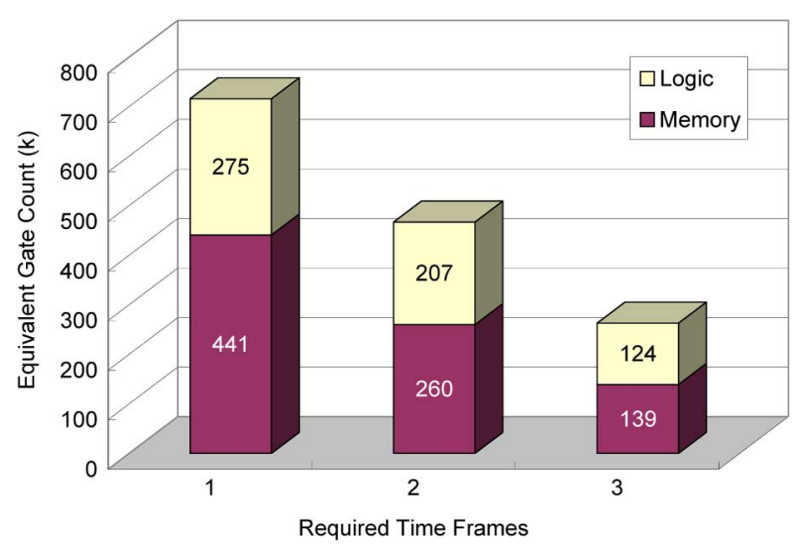

Fig. 18. Equivalent gate counts for different required time frames.

(ECI) [33], on CRISP. Note that, among these four methods, $\mathrm{ECI}$ is the most complex one with more arithmetic operations and memory requirement.

The mapped hardware resources and processing performance are shown in Table VII. The hardware resource of the prototype CRISP chip is enough for mapping all of these color interpolation methods with different cost and performance in one time frame. Since the latency of the critical module for ECI method is two cycles, the output data rate is 172 Mpixles/s, which is half of the other three methods. From this example, it shows that CRISP contains high flexibility for different image pipeline algorithms, which can be mapped with different data rates according to algorithmic complexity.

\section{B. Offline Flexibility: Scalable Hardware Resource and Processing Time Tradeoff}

One example for offline flexibility is shown here, where an image signal-processing pipeline similar to that in Fig. 15 is implemented. We set different processing speed requirements in terms of the number of time frames, where one, two, and three time frames are considered. Then, the minimum required hardware cost is determined, including line buffers and other RSPEs. The equivalent gate counts of both logic part and memory part are shown in Fig. 18. This shows that, if fewer time frames are needed for higher processing speed, this will require more hardware cost. We can also see that, for different execution performance, the memory size increases faster than the logic size when fewer time frames are available. This indicates that on-chip local memory may dominate the total chip size for image processors with higher performance. From this example, it demonstrates that CRISP contains offline flexibility for different application requirements. Before the chip fabrication, designers can decide the devotion numbers of modules based on different time-space tradeoff considerations.

\section{CONCLUSION}

In this paper, a novel CRISP architecture for image signal-processing pipelines of DSCs and camcorders is proposed. By the observation of the different properties of the preview and picture-taking modes of DSCs and the algorithmic similarity of image-processing tasks, new concepts of coarse-grained reconfigurable image stream processors are proposed to devote low-cost hardware for requirements in the preview mode and add some hardware resources for higher flexibility and processing capability for the picture-taking mode to support more complex algorithms. After that, the hardware architecture of CRISP is proposed with RSPEs and reconfigurable interconnection. Six kinds of RSPEs are proposed to cover most of the image-processing tasks: color interpolation RSPEs and downsampler RSPEs are proposed for various color interpolation algorithms and downsampling with different scaling ratios; local memory RSPEs, pixel-based operation RSPEs, and multiplication and accumulation RSPEs are proposed for general image-processing operations; ALU RSPE is designed for other general operations. In addition, reconfigurable interconnection is proposed with the same input and output protocols for each module. A prototype chip of CRISP is fabricated with TSMC $0.18-\mu \mathrm{m} 1 \mathrm{P} 6 \mathrm{M}$ CMOS process via chip implementation center (CIC). With the core size of $5 \mathrm{~mm}^{2}$ and power consumption of $218 \mathrm{~mW}$ at the working frequency of $115 \mathrm{MHz}, \mathrm{CRISP}$ can achieve the requirements of high-end 10-Mpixel-scale DSCs and high-definition video camcoders. The implementation results also demonstrate that CRISP has similar computation efficiency to ASICs for preview and can accelerate the execution of typical image pipelines dramatically, whose processing speed is over 218 times faster than Trimedia TM1300 media processor and over 83 times faster than TMS320C64x DSP, which shows the concept of CRISP is successfully proved. It also shows that CRISP owns multilevel reconfigurability, including the number of each kind of modules determined before fabrication, and online reconfigurability of the interconnection order between modules and the functionality inside each module.

Although the efficiency of CRISP outperforms existing solutions for image signal processing, it still has some drawbacks. First, CRISP is optimized only for the tasks of image signalprocessing pipelines and may not be efficient for other imageprocessing tasks, such as image compression and image analysis. Therefore, CRISP can only be viewed as an accelerator for image signal-processing pipelines for DSCs and camcorders, which means other hardware components, such as a generalpurpose processor, may be still required. It can also integrated 
with DSP to form a more powerful image processor. In addition, since the compiler or other related development tools are not developed, the contexts of CRISP for RSPEs and reconfigurable interconnection are designed manually. It is not easy for software programmers to program CRISP and may still need help from hardware engineers. These issues will be considered in our future work.

\section{ACKNOWLEDGMENT}

The authors would like to thank the Chip Implementation Center (CIC) for chip fabrication.

\section{REFERENCES}

[1] E. Fossum, "Digital camera system on a chip," IEEE Micro, vol. 18, no. 3, pp. 8-15, May-Jun. 1998.

[2] W. Rabadi, R. Talluri, K. Illgne, J. Liang, and Y. Yoo, "Programmable DSP platform for digital still cameras," in Proc. 1999 IEEE Int. Conf. Acoust., Speech, Signal Process. (ICASSP99), Mar. 1999, pp. $2235-2238$.

[3] W. Rabadi, R. Talluri, K. Illgner, J. Liang, and Y. Yoo, "Programmable DSP Platform for Digital Still Cameras," Application Rep., Apr. 2000, TI.

[4] N. Nakano, R. Nishimura, H. Sai, A. Nishizawa, and H. Komatsu, "Digital still camera system for megapixel CCD," IEEE Trans. Consumer Electron., vol. 44, no. 3, pp. 581-586, Aug. 1998.

[5] H. Zen, T. Koizumi, H. Yamamoto, and I. Kimura, "A new digital signal processor for progressive scan CCD," IEEE Trans. Consumer Electron., vol. 4, no. 2, pp. 289-296, May 1998.

[6] R. Zhou, X. Chen, F. Liu, J. He, T. Liao, Y. Su, J. Ye, Y. Qin, X. Yi, and Z. Hong, "System-on-chip for mega-pixel digital camera processor with auto control functions," in Proc. Int. Conf. ASIC, Oct. 2003, pp. 894-897.

[7] D. Talla, C.-Y. Hung, R. Talluri, F. Brill, D. Smith, D. Brier, B. Xiong, and D. Huynh, "Anatomy of a portable digital mediaprocessor," IEEE Micro, vol. 24, no. 2, pp. 32-39, Mar.-Apr. 2004.

[8] "TMS320DSC21-A High-Performance, Programmable, Single Chip Digital Signal Processing Solution to Digital Still Cameras" Texas Instruments, 2006 [Online]. Available: http://focus.ti.com/lit/ml/ spry086/spry086.pdf

[9] D. Talla, R. Austen, D. Brier, C.-Y. Hung, D. Huynh, D. Smith, B. Xiong, R. Talluri, and F. Brill, "TMS320DM310-A portable digital media processor," in Proc. IEEE HOT Chips, Aug. 2003, pp. 144-151.

[10] R. Hartenstein, "Reconfigurable computing: A new business model-and its impact on SoC design," in Proc. Euromicro Symp. Digital Syst., Design, Sep. 2001, pp. 103-110.

[11] H. Singh, M.-H. Lee, G. Lu, F. Kurdahi, N. Bagherzadeh, and E. C. Filho, "MorphoSys: An integrated reconfigurable system for data-parallel and computation-intensive applications," IEEE Trans. Comput., vol. 49, no. 5, pp. 465-481, May 2000.

[12] J. Rose, A. E. Gamal, and A. Sangiovanni-Vincentelli, "Architecture of field-programmable gate arrays," Proc. IEEE, vol. 81, no. 7, pp. 1013-1029, Jul. 1993.

[13] R. Hartenstein, "Coarse grain reconfigurable architectures," in Proc. Asia South Pacific Design Autom. Conf., Jan. 2001, pp. 564-569.

[14] C. Ebeling, D. C. Cronquist, and P. Franklin, "RaPiD reconfigurable pipelined datapath," in Proc. 6th Int. Workshop Field-Programmable Logic and Compilers, Aug. 1996, pp. 126-135.

[15] S. Goldstein, H. Schmit, M. Budiu, S. Cadambi, M. Moe, and R. Taylor, "PipeRench: A reconfigurable architecture and compiler," IEEE Computer, vol. 33, no. 4, pp. 70-77, Apr. 2000.

[16] E. Mirsky and A. DeHon, "MATRIX: A reconfigurable computing architecture with configurable instruction distribution and deployable resources," in Proc. IEEE Symp. FPGAs for Custom Computing Machines, Apr. 1996, pp. 157-166.

[17] T. J. Callahan, J. R. Hauser, and J. Wawrzynek, "The Garp architecture and C compiler," IEEE Computer, vol. 33, no. 4, pp. 62-69, Apr. 2000.

[18] E. Waingold, M. Taylor, D. Srikrishna, V. Sarkar, W. Lee, V. Lee, J. Kim, M. Frank, P. Finch, R. Barua, J. Babb, S. Amarasinghe, and A. Agarwal, "Baring it all to software: RAW machines," IEEE Computer, vol. 30, no. 9, pp. 86-93, Sep. 1997.
[19] T. Miyamori and K. Olukotun, "REMARC: Reconfigurable multimedia array coprocessor," in Proc. ACM/SIGDA FPGA98, Feb. 1998, p. 261.

[20] A. Marshall, T. Stansfield, I. Kostarnov, J. Vuillemin, and B. Huichings, "A reconfigurable arithmetic array for multimedia applications," in Proc. ACM/SIGDA FPGA99, Feb. 1999, pp. 135-143.

[21] R. Soares, A. Azevedo, and I. S. Silva, "X4CP32: A coarse grain general purpose reconfigurable microprocessor," in Proc. Int. Parallel Distrib. Process. Symp., Apr. 2003.

[22] N. Suzuki, S. Kurotaki, M. Suzuki, N. Kaneko, Y. Yamada, K. Deguchi, Y. Hasegawa, and H. Amano, "Implementing and evaluating stream applications on the dynamically reconfigurable processor," in Proc. IEEE Symp. Field-Programmable Custom Computing Machines, Apr. 2004, pp. 328-329.

[23] “XPP-III Processor Overview,” PACT XPP Technologies Inc. , 2006 [Online]. Available: http://www.pactxpp.com

[24] K. Tanigawa, T. Hironaka, A. Kojima, and N. Yoshida, "PARS architecture: A reconfigurable architecture with generalized execution model - design and implementation of its prototype processor," IEICE Trans. Inf. Syst., vol. E86-D, no. 5, pp. 830-840, May 2003.

[25] B. Mei, S. Vernalde, D. Verkest, H. D. Man, and R. Lauwereins, "ARDES: An architecture with tightly coupled VLIW processor and coarse-grained reconfigurable matrix," in Proc. Field-Programmable Logic Applic., 2003, pp. 61-70.

[26] J. Adams, K. Parulski, and K. Spaulding, "Color processing in digital cameras," IEEE Micro, vol. 18, no. 6, pp. 20-30, Nov. 1998.

[27] P. W. Wong, D. Tretter, C. Herley, N. Moayeri, and R. Lee, "Image processing considerations for digital photography," in Proc. Compcon'97., Feb. 1997, pp. 280-285.

[28] R. Ramanath, W. E. Snyder, Y. Yoo, and M. S. Drew, "Color image processing pipeline: A general survey of digital still camera processing," IEEE Signal Process. Mag., vol. 22, no. 1, pp. 34-43, Jan. 2005.

[29] B. Bayer, "Color Imaging Array," U.S. Patent 3971 065, Jul. 20, 1976.

[30] J. E. Adams, "Interactions between color plane interpolation and other image processing functions in electronic photography," in Proc. SPIE, Mar. 1995, vol. 2416, pp. 144-151.

[31] R. H. Hibbard, "Apparatus and Method for Adaptively Interpolating a Full Color Image Utilizing Luminance Gradients," U.S. Patent 5382 976, Jan. 17, 1995.

[32] J. F. Hamilton and J. E. Adams, "Adaptive Color Plan Interpolation in Single Sensor Color Electronic Camera," U.S. Patent 5629 734, May 13, 1997.

[33] S.-C. Pei and I.-K. Tam, "Effective color interpolation in ccd color filter arrays using signal correlation," IEEE Trans. Circuits Syst. Video Technol., vol. 13, no. 6, pp. 503-513, Jun. 2003.

[34] J. J. E. Adams, "Design of practical color filter array interpolation algorithms for digital cameras," in Proc. SPIE, Mar. 1997, vol. 3028, pp. $117-125$, SPIE.

[35] S.-C. Hsia, M.-H. Chen, and P.-S. Tsai, "VLSI implementation of lowpower high-quality color interpolation processor for CCD camera," IEEE Trans. Very Large-Scale Integr. (VLSI) Syst., vol. 14, no. 4, pp. 361-369, Apr. 2006

[36] P. A. Ruetz and R. W. Brodersen, "Architectures and design techniques for real-time image-processing IC's," IEEE J. Solid-State Circuits, vol. sc-22, no. 2, pp. 233-250, Apr. 1987.

[37] L. Lucas, "High speed low cost TM1300 Trimedia enhanced PCI VLIW mediaprocessor," in Proc. HOT CHIPS 11, 1999.

[38] S. Agarwala, P. Koeppen, T. Anderson, A. Hill, M. Ales, R. Damodaran, L. Nardini, P. Wiley, S. Mullinnix, J. Leach, A. Lell, M. Gill, J. Golston, D. Hoyle, A. Rajagopal, A. Chachad, M. Agarwala, R. Castille, N. Common, J. Apostol, H. Mahmood, M. Krishnan, D. Bui, Q.-D. A. P. Groves, N. Luong, N. Nagaraj, and R. Simar, "A 600 MHz VLIW DSP," in Dig. Tech. Papers IEEE Int. Solid-State Circuits Conf. (ISSCC2002), Feb. 2002, vol. 2, pp. 38-390.

[39] B. Mei, S. Vernalde, D. Verkest, H. D. Man, and R. Lauwereins, "IMAP-CE: A 51.2 GOPS video rate image processor with 128 VLIW processing elements," in Proc. Int. Conf. Image Process., Oct. 2001, vol. 3, pp. 294-297.

[40] A. Abbo, R. Kleihorst, V. Choudhary, L. Sevat, P. Wielage, S. Mouy, and M. Heijligers, "XETAL-II: A 107 GOPS, $600 \mathrm{~mW}$ massively-parallel processor for video scene analysis," in Dig. Tech. Papers IEEE Int. Solid-State Circuits Conf. (ISSCC2007), Feb. 2007, pp. 270-602.

[41] U. Kapasi, S. Rixner, W. Dally, B. Khailany, J. H. Ahn, P. Mattson, and J. Owens, "Programmable stream processors," Computer, vol. 36, no. 8, pp. 54-62, Aug. 2003.

[42] B. Khailany, T. Williams, J. Lin, E. Long, M. Rygh, D. Tovey, and W. J. Dally, "A programmable 512 GOPS stream processor for signal, image, and video processing," in Dig. Tech. Papers IEEE int. SolidState Circuits Conf. (ISSCC2007), Feb. 2007, pp. 272-602. 


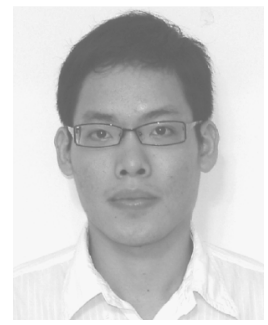

Jason C. Chen received the B.S. degrees in electronics engineering from National Chiao-Tung University (NCTU), Hsinchu, Taiwan, R.O.C., and the M.S. degrees from the Graduate Institute of Electronics Engineering (GIEE), National Taiwan University (NTU), Taipei, Taiwan, R.O.C., in 2004 and 2006, respectively.

From 2004 to 2006, he was with Media IC and System Laboratory, GIEE, NTU, where his research interests included video coding technology, image processing, reconfigurable computing, processor architecture design, and VLSI implementation.

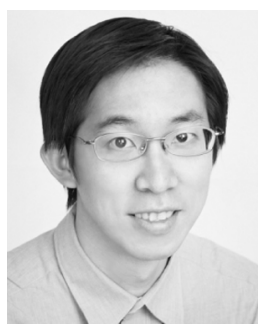

Shao-Yi Chien (M'04) received the B.S. and Ph.D. degrees in electrical engineering from National Taiwan University (NTU), Taipei, Taiwan, R.O.C., in 1999 and 2003, respectively.

From 2003 to 2004, he was a Member of Research Staff with Quanta Research Institute, Tao Yuan Shien, Taiwan, R.O.C. In 2004, he joined the Graduate Institute of Electronics Engineering and Department of Electrical Engineering, NTU, as an Assistant Professor. His research interests include video segmentation algorithm, intelligent video coding technology, image processing, computer graphics, and associated VLSI architectures. 